\title{
Plk1 interacts with RNF2 and promotes its ubiquitin-dependent degradation
}

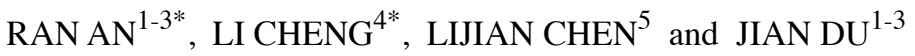 \\ ${ }^{1}$ Department of Biochemistry and Molecular Biology, School of Basic Medical Sciences; \\ ${ }^{2}$ Anhui Province Key Laboratory of Pathogen Biology; ${ }^{3}$ Anhui Key Laboratory of Zoonoses; \\ ${ }^{4}$ Department of Orthopaedics, The Second Affiliated Hospital of Anhui Medical University; \\ ${ }^{5}$ Department of Anesthesiology, The First Affiliated Hospital, Anhui Medical University, \\ Hefei, Anhui 230032, P.R. China
}

Received September 20, 2017; Accepted March 12, 2018

DOI: $10.3892 /$ or.2018.6326

\begin{abstract}
Ring finger protein 2 (RNF2), also known as RING2 or RING1B, displays oncogenic functions in different types of cancers, yet, the function of RNF2 during mitosis has not been evaluated. A yeast two-hybrid screen was undertaken using a human HeLa cDNA library to explore and identify proteins that interact with RNF2. Several positive clones, including Polo-like kinase 1 (Plk1), a critical regulator of mitosis, were identified. The interaction between RNF2 and Plk1 was confirmed using a $\beta$-galactosidase and growth test in selective media, in vitro glutathione $S$-transferase pull-down, and in vivo immunoprecipitation assays. Moreover, we confirmed that RNF2 co-localized with Plk1 at mitotic chromosomes in the prometaphase and metaphase using an immunofluorescence assay. In addition, our results revealed that Plk1 kinase activity was required for ubiquitin-dependent degradation of RNF2. These findings provide a new clue for understanding the function of RNF2 during mitotic regulation and tumorigenesis.
\end{abstract}

\section{Introduction}

Polo-like kinase 1 (Plk1) is an evolutionarily conserved serine/threonine protein kinase and a key regulator of mitosis that is required for kinetochore-microtubule attachments, centrosome maturation, chromosome dynamics, chromosome

Correspondence to: Dr Lijian Chen, Department of Anesthesiology, The First Affiliated Hospital, Anhui Medical University, Hefei, Anhui 230032, P.R. China

E-mail: chenlijian77@126.com

Professor Jian Du, Department of Biochemistry and Molecular Biology, School of Basic Medical Sciences, Anhui Medical University, Hefei, Anhui 230032, P.R. China

E-mail: dujane@163.com; dujian@ahmu.edu.cn

${ }^{*}$ Contributed equally

Key words: Plk1, RNF2, protein interaction, degradation segregation, spindle function and cytokinesis (1-3). Full length Plk1 consists of the N-terminus kinase domain (KD) and the C-terminus Polo-box domain (PBD) (4). Its functions are executed by binding and phosphorylating proteins through its two domains. The different subcellular location of Plk1 is based on the PBD and phosphorylation of Thr210 $(5,6)$. Diverse functions of the Polo-like kinases (PLKs) have been reported at different stages of the cell cycle. An association between PLKs and the centrosome is implicated in regulating mitotic entry. Sudden Plk1 activation during late G2 depends on cyclin $\mathrm{A} / \mathrm{Cdk}$ activity levels and induces phosphorylation of Cdc25C1 before mitotic entry (7). Plk1 phosphorylates BubR1 in mitotic cells, and this phosphorylation is associated not only with spindle checkpoint signaling but also with stable microtubule-kinetochore attachment, probably by generating the force that pulls each centrosome towards the two spindle poles $(8,9)$. Plk1 interacts with the key mitotic kinase Aurora B. Aurora B-dependent phosphorylation of the Plk1 activation loop at the kinetochore regulates its function (10). Additionally, recruitment of Aurora B to the centromeres depends partly on Plk1-dependent phosphorylation of survivin and haspin. Therefore, Plk1 is a critical mitotic kinase during the cell cycle.

Ring finger protein 2 (RNF2), also known as RING2 or RING1B, was first identified as an interactor with Bmil, a group II polycomb group (PcG) protein. RNF2 acts as a ubiquitin E3 ligase to mono-ubiquitinate histone H2A (11-13). Moreover, RNF2 is involved in regulating different biological processes by distinct molecular mechanisms. For example, RNF2 promotes MDM2-mediated p53 ubiquitination, and overexpression of RNF2 increases the half-life of MDM2 and inhibits its ubiquitination (14). Furthermore, one study revealed that negative regulation of $\mathrm{p} 53$ by RNF2 promoted tumor development in selective cancer cell types (15). RNF2 is ubiquitously expressed in human tissues and is amplified or overexpressed in many human tumors, such as ovarian, breast and pancreatic cancer (16-18). RNF2 is considered to be a prognostic biomarker and potential therapeutic target for these cancer types, as high expression of RNF2 is positively correlated with tumor progression and shortened survival. However, the extensive biochemical characterization of RNF2 in mitotic regulation has not been elucidated. 
In the present study, we identified for the first time that Plk1 interacts with RNF2 using a yeast two-hybrid screen. We sought to elucidate the relationship between Plk1 and RNF2. Our results demonstrated that RNF2 co-localized with Plk1 at mitotic chromosomes in the prometaphase and metaphase. In addition, Plk1 kinase activity was required for the ubiquitin-dependent degradation of RNF2. These findings provide a new clue to our understanding of the function of RNF2 in mitotic regulation and tumorigenesis.

\section{Materials and methods}

Cell culture, reagents and plasmids. HeLa and 293T cells (ATCC, Manassas, VA, USA) were grown at $37^{\circ} \mathrm{C}$ in a $5 \% \mathrm{CO}_{2}$ atmosphere in Dulbecco's modified Eagle's medium (DMEM; HyClone Laboratories Inc.; Thermo Fisher Scientific, Inc., Waltham, MA, USA), supplemented with $10 \%$ fetal bovine serum (FBS; HyClone Laboratories Inc.; Thermo Fisher Scientific, Inc.), $100 \mu \mathrm{g} / \mathrm{ml}$ streptomycin and $100 \mu \mathrm{g} / \mathrm{ml}$ penicillin. The cells were synchronized at the G1/S phase with a $250 \mathrm{mM}$ thymidine (Sigma-Aldrich; Merck KGaA, Darmstadt, Germany) block. The plasmid vectors 3x FLAG (Sigma-Aldrich; Merck KGaA), pEGFP-C2 (BD Biosciences, San Jose, CA, USA), pGBKT7 (Clontech Laboratories, Inc., Palo Alto, CA, USA) and pGADT7 (Clontech Laboratories, Inc.) were used to generate mammalian and yeast expression constructs carrying Plk1 or RNF2. Plk1 or RNF2 was cloned into the bacterial expression plasmids pET-28a (Novagen, Wilmington, DE, USA) and pGEX-6P-1 (GE Healthcare, Parsippany, NJ, USA). MG132 (EMD Millipore, Bedford, MA, USA), anti-RNF2 antibody (cat. no. ab101273) and anti-Plk1 antibody (cat. no. ab17057) were both purchased from Abcam (Cambridge, MA, USA). GFP (cat. no. G1546) and FLAG (cat. no. F1804) antibodies were purchased from Sigma-Aldrich (Merck KGaA).

Yeast two-hybrid analysis. The specificity of the interaction between Plk1 and RNF2 was verified by yeast two-hybrid. RNF2 was fused to pGBKT7 to generate the BD-RNF2 vector. Plk1 was fused to pGADT7 to generate the AD-Plk1 vector. Yeast two hybrid screening and yeast two hybrid co-transformation assays were performed following the manufacturer's instructions for the Yeast transformation system 2 (Clontech Laboratories, Inc.). The yeast was detected on yeast dropout medium lacking tryptophan, leucine and histidine.

Glutathione S-transferase (GST) pull-down assay. Purified RNF2-His was incubated with Glutathione Sepharose 4B beads carrying the fusion proteins (PLK1-GST or GST) at $4^{\circ} \mathrm{C}$ for $4 \mathrm{~h}$. After washing with pre-cooled phosphate-buffered saline (PBS) containing $1 \%$ Triton $\mathrm{X}-100$, the beads were fixed in a sample loading buffer at $100^{\circ} \mathrm{C}$ for $10 \mathrm{~min}$ and then subjected to sodium dodecyl sulfate-polyacrylamide gel electrophoresis (SDS-PAGE) followed by immunoblot assay.

Immunoblot assay and immunoprecipitation. 293T or HeLa cells transformed with the corresponding plasmids were harvested and resuspended in lysis buffer $(\mathrm{pH} 7.4,50 \mathrm{mM}$ HEPES, $150 \mathrm{mM} \mathrm{NaCl}, 2$ mM EGTA, 0.1\% Triton X-100, $1 \mathrm{mM}$ phenylmethylsulfonyl fluoride, $10 \mathrm{~g} / \mathrm{ml}$ leupeptin and $10 \mathrm{~g} / \mathrm{ml}$ pepstatin A) containing a protease inhibitor mixture (Sigma-Aldrich; Merck KGaA). After separation by SDS-PAGE, the proteins were transferred to nitrocellulose membranes. After blocking in TBST buffer with 5\% non-fat milk, the membranes were probed with the corresponding antibodies, and developed using an enhanced chemiluminescence reagent. The cell lysates were purified using anti-FLAG M2 affinity gel at $4^{\circ} \mathrm{C}$ for $4 \mathrm{~h}$ with rotation for immunoprecipitation. After washing with lysis buffer and PBS, the immunoprecipitants were eluted by boiling with a sample loading buffer at $100^{\circ} \mathrm{C}$ for $10 \mathrm{~min}$ and then subjected to SDS-PAGE followed by immunoblot assay.

Immunofluorescence. HeLa cells were harvested and plated on poly-L-lysine-coated sterile glass slides. Then, the cells were fixed in $4 \%$ paraformaldehyde for $15 \mathrm{~min}$ and permeabilized with PBS containing $0.1 \%$ Triton X-100 for 15 min. After washing in PBS, the coverslips were blocked with PBS containing 5\% bovine serum albumin (BSA). Subsequently, the coverslips were incubated with anti-Plk1 (1:100; cat. no. ab17057; Abcam) and anti-RNF2 (1:100; cat. no. ab101273; Abcam) overnight at $4^{\circ} \mathrm{C}$ and incubated with the secondary antibodies FITC-conjugated goat anti-mouse IgG (1:500; cat. no. F-2761; Thermo Fisher Scientific, Inc.) and rhodamine-conjugated goat anti-rabbit IgG (1:500; cat. no. R-6394; Thermo Fisher Scientific, Inc.) and DAPI dye. Images were captured using an Olympus BX60 upright fluorescence microscope (Olympus Corp., Tokyo, Japan) with identical acquisition parameters for each experiment.

\section{Results}

Identification of Plk1 as a RNF2-binding protein. To identify host proteins targeted by RNF2, the yeast two-hybrid screen was undertaken to screen the human HeLa cDNA library. Surprisingly, Plk1, an important mitotic regulator was found among the positive clones. To confirm the interaction between Plk1 and RNF2, we co-transformed AH109 yeast competent cells with Plk1-pGADT7 and RNF2-pGBKT7. The results demonstrated that Plk1 interacted with RNF2 (Fig. 1A). To further confirm this interaction, we performed a GST pull-down assay in vitro using recombinant RNF2-His and Plk1-GST proteins expressed in E. coli. Plk1-GST, but not GST, was able to pull down RNF2-His, demonstrating that Plk1 directly bound to RNF2 (Fig. 1B). To further confirm this result, we co-transfected Plk1-FLAG and RNF2-GFP into 293T cells for an immunoprecipitation assay using an anti-FLAG antibody. The results revealed that RNF2-GFP was pulled down by the FLAG antibody via Plk1-FLAG (Fig. 1C). To test if endogenous RNF2 formed a complex with Plk1 in 293T cells, we carried out an immunoprecipitation with RNF2 antibody and control IgG. The result indicated that endogenous RNF2 interacted with Plk1, suggesting that RNF2 interacts with Plk1 in vivo (Fig. 1D).

RNF2 and Plk1 are co-localized in HeLa cells during mitosis. Dynamic localization during mitosis is one of the most striking aspects of Plk1. To evaluate if there is specific co-localization and the role of the interaction between RNF2 and Plk1 during mitosis, we conducted immunofluorescence 
A

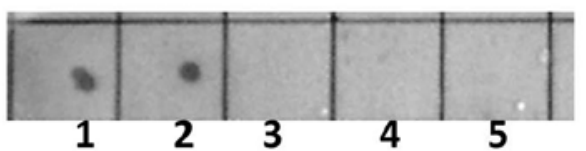

1. BD-RNF2+AD-PIk1

2. BD-53+AD-T (positive control)

3. BD-lam+AD-T (negative control)

4. BD-RNF2

5. AD-PIk1

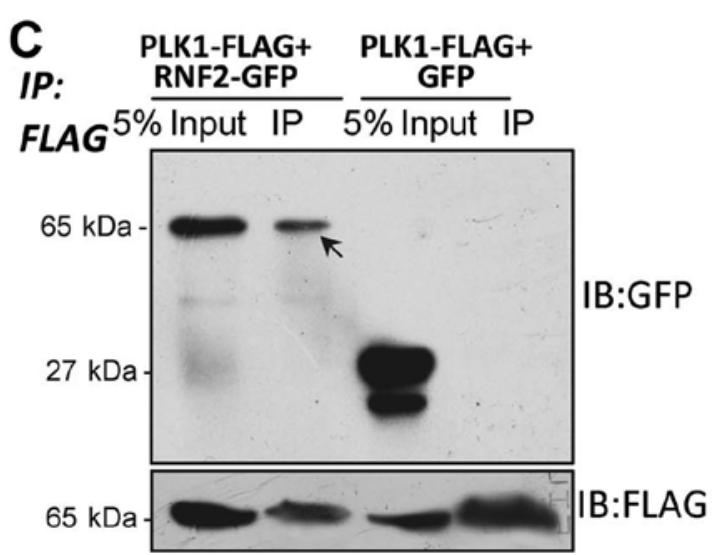

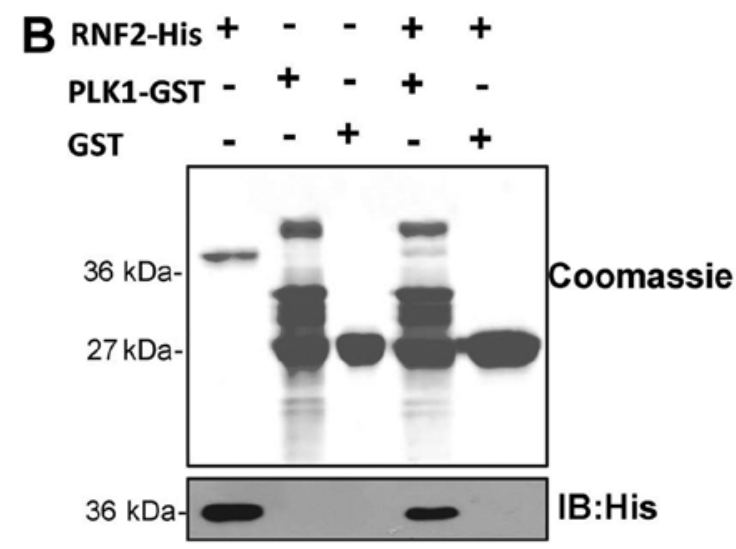

D

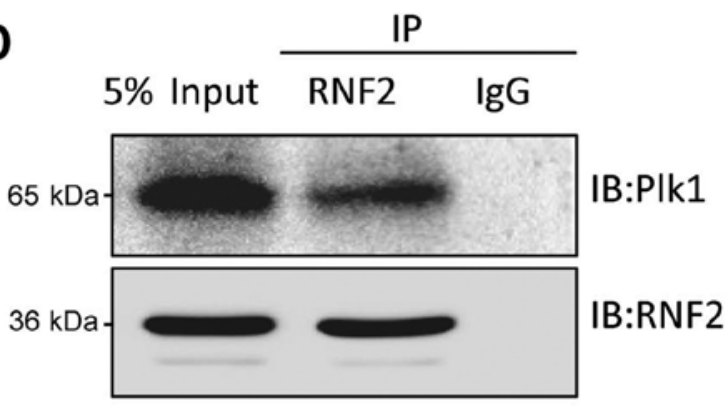

Figure 1. Identification of Plk1 as a RNF2-interacting protein. (A) The interaction between RNF2 and Plk1 was identified by a yeast two-hybrid system. Screening plasmids expressing RNF2 fused to the GAL4 DNA-binding domain were co-transformed with a plasmid expressing Plk1 fused to the GAL4 transactivation domain. Interactions were detected through growth on medium containing $\mathrm{x}$ - $\alpha$-gal and lacking Ade, Trp, Leu and His. BD-53 and AD-T were co-transformed into yeast cells as positive controls, and BD-lam and AD-T were co-transformed into yeast cells as negative controls. The results reveal that Plk1 indeed interacts with RNF2. (B) Confirmation of Plk1 binding to RNF2 using in vitro pull-down assays. Plk1-GST purified on GST beads was used as an affinity matrix for absorbing RNF2-His. The SDS-PAGE gel was stained with Coomassie brilliant blue (upper panel) and subsequently blotted with anti-His antibody (lower panel). (C) 293T cells co-transfected with Plk1-FLAG and RNF2-GFP or control GFP vector were immunoprecipitated with anti-FLAG. Starting fractions (Input) and immunoprecipitates (IP) were analyzed by SDS-PAGE and western blotting using GFP and FLAG antibodies. (D) Immunoprecipitation of Plk1 and RNF2. HeLa cell lysates were incubated with an anti-RNF2 antibody or a non-specific IgG and the protein A/G-Sepharose beads. The expression of Plk1 and RNF2 was detected by immunoblotting with anti-Plk1 and anti-RNF2 antibody, respectively.

studies to assess the localization of the two proteins at different mitotic stages in HeLa cells. Notably, we found a defined co-localization of RNF2 and Plk1 at the mitotic chromosomes in the prometaphase and metaphase during mitosis (Fig. 2). RNF2 was diffusely localized at the nucleus during interphase, which is consistent with its known role in transcriptional regulation. Plk1, as expected, was found scattered in the cytoplasm (Fig. 2A). As the cells entered prometaphase, Plk1 began to localize to the spindle poles and the kinetochores. RNF2 demonstrated increasing localization at the mitotic chromosome that overlapped Plk1 at the kinetochores (Fig. 2B). Plk1 was concentrated at the kinetochores in the metaphase. The examination of RNF2 labeling in the same cells revealed typical mitotic chromosome localization. Thus, RNF2 and Plk1 co-localized at the mitotic chromosomes during the prometaphase and metaphase (Fig. 2C). Furthermore, as chromosome segregation occurred in the anaphase and telophase, Plk1 was maintained at the mid-body and RNF2 extended into the nucleus of each sister cell (Fig. 2D and E). These results indicate that RNF2 and Plk1 are co-localized at mitotic chromosomes during mitotic progression.
Plk1 kinase activity is responsible for degradation of RNF2. We demonstrated that Plk1 is a bona fide RNF2-interacting protein, thus we aimed to address the functional relevance of this interaction. We overexpressed GFP-tagged wild-type Plk1 in HeLa cells and subsequently monitored the relative levels of endogenous RNF2 in these cells via western blotting. The result revealed that overexpression of wild-type Plk1 reduced the level of RNF2 in a dose-dependent manner (Fig. 3A and B). Next, we aimed to ascertain whether Plk1 kinase activity is essential for degradation of RNF2; thus, we overexpressed GFP-tagged kinase-deficient Plk1 in HeLa cells and detected the relative levels of endogenous RNF2. Notably, overexpression of kinase-deficient Plk1 did not affect the level of endogenous RNF2 (Fig. 3C and D). Thus, we concluded that Plk1 kinase activity is responsible for RNF2 degradation.

Plk1 interacts with RNF2 and promotes its proteasomeubiquitin-dependent degradation. Western blot analysis indicated that wild-type Plk1 reduced the level of RNF2, but kinase-deficient Plk1 failed to do so. To explore whether Plk1 reduces RNF2 mRNA, we performed a quantitative reverse transcription-polymerase chain reaction (qRT-PCR) analysis. 
A
Interphase
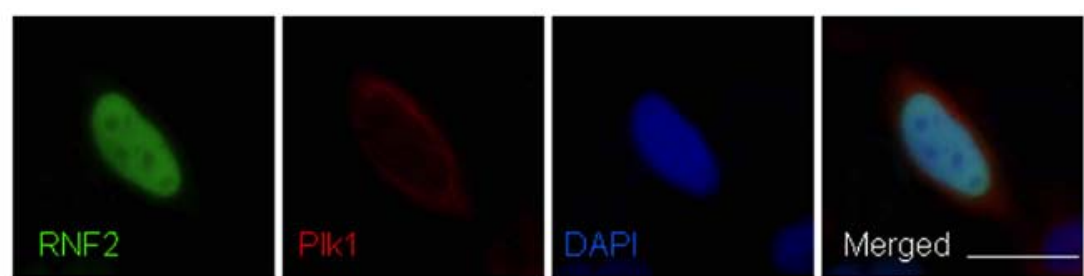

B

Prometaphase
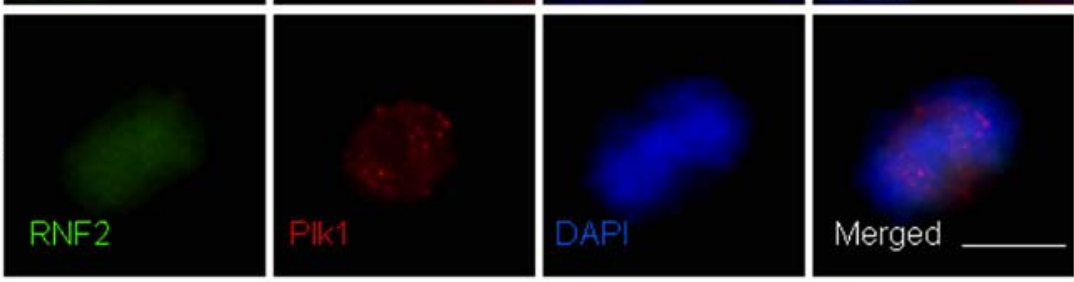

C
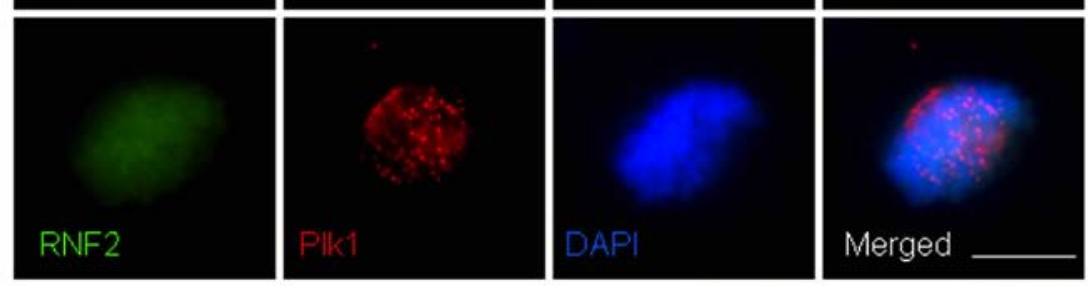

D

Anaphase
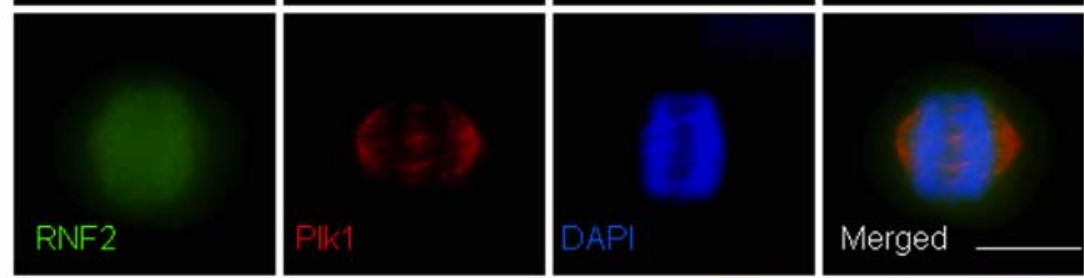

E
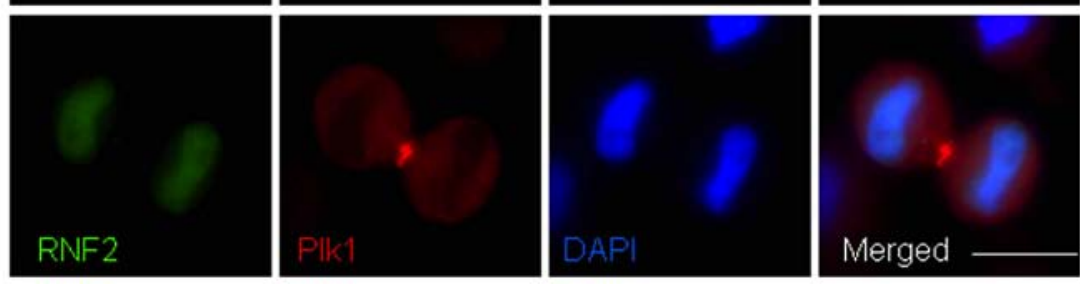

Figure 2. Co-localization of Plk1 and RNF2 during mitotic progression by immunofluorescence staining. Subcellular localization of Plk1 and RNF2 in HeLa cells using immunofluorescent staining. HeLa cells were evaluated at the interphase (A), prometaphase (B), metaphase (C), anaphase (D) and telophase (E). The cells were stained with anti-Plk1 antibody (green), anti-RNF2 antibody (red) and chromosomes (blue). Scale bars, $10 \mu \mathrm{m}$.

The results revealed that the RNF2 mRNA level did not change significantly after overexpressing Plk1, suggesting a Plk1-dependent reduction in the RNF2 protein level (Fig. 4A). Moreover, since overexpression of wild-type Plk1 resulted in lowering RNF2 protein levels, we anticipated that Plk1 knockdown may create an opposite effect. The results showed that Plk1 knockdown resulted in a significant increase in the RNF2 level (Fig. 4B). To determine whether Plk1-induced RNF2 degradation was proteasome-ubiquitin-dependent, 293T cells were cotransfected with control or Plk1 siRNA and myc-tagged ubiquitin. The proteasome inhibitor MG132 was used to block degradation of possibly ubiquitinated RNF2. The results demonstrated that ubiquitination of RNF2 decreased significantly in response to depleted Plk1 in the presence of MG132, suggesting that Plk1 is required for ubiquitin-dependent degradation of RNF2 (Fig. 4C). In addition, to further test the possible effect of Plk1 kinase on the ubiquitin-dependent degradation of RNF2, we repeated the above experiment using wild-type or kinase-deficient Plk1 plasmids. As expected, ubiquitination of RNF2 was increased significantly by Plk1-WT-GFP, compared with that of Plk1-KD-GFP in the presence of MG132 (Fig. 4D). Therefore, we concluded that
Plk1-dependent decrease in RNF2 protein level is mediated through proteasome-ubiquitin-dependent degradation.

\section{Discussion}

The polycomb group $(\mathrm{PcG})$ of proteins are epigenetic transcriptional regulators that repress numerous developmental regulators, including two repressive complexes, called PRC1 and PRC2. RNF2, also known as RING2 or RING1B, is a member of the PRC1 complex. Previous studies have reported that RNF2 directly associates with Bmi1, Cbx family proteins, Mel18, M33 and Phc1, forming the core of the PRC1 complex (18-20). The complex varies in its association with mitotic chromosomes. Live-cell imaging and genome-wide sequencing demonstrates that most PRC1 proteins dissociate from mitotic chromosomes, whereas a quantitative subpopulation of PRC1 proteins remain associated with mitotic chromosomes in Drosophila. Recent studies have shown that canonical PRC1 exhibits various capacities of association with mitotic chromosomes and that $\mathrm{Cbx} 2$ is required to recruit PRC1 to mitotic chromosomes (20-22). Previous studies have revealed that Plk1 phosphorylates numerous mitotic proteins, 

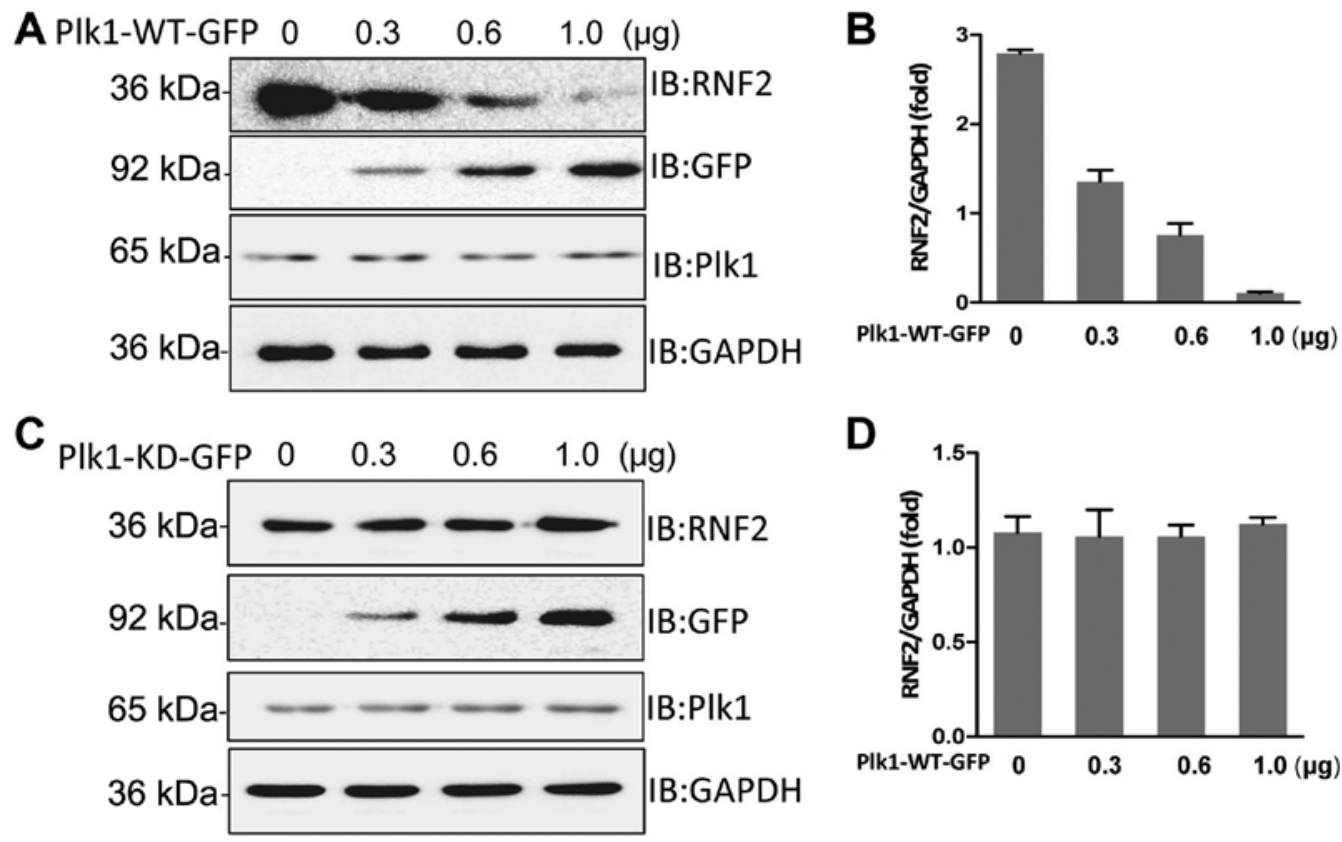

Figure 3. Plk1 kinase activity is responsible for RNF2 degradation. (A) Lysates of 293T cells were transiently co-transfected with the indicated volumes of the GFP-tagged wild-type Plk1 expression vectors, and then the levels of endogenous RNF2 were detected through western blotting with the indicated antibodies. (B) The levels of RNF2 in A were quantified using the mean ratio of RNF2 to GAPDH for triplicate results. Error bars represent the mean \pm SD of triplicates. (C) Lysates of 293T cells transiently co-transfected with the indicated volumes of the GFP-tagged kinase-deficient Plk1 expression vectors, then the levels of endogenous RNF2 were detected through western blotting with the indicated antibodies. (D) The RNF2 levels in C were quantified using the mean ratio of RNF2 to GAPDH for triplicate results. Error bars represent the mean \pm SD of triplicates.

A

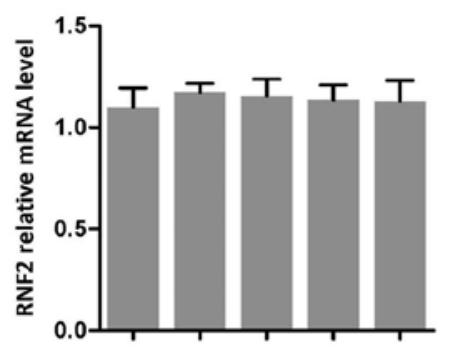

GFP vector $(\mu \mathrm{gg}) \quad 1.0 \quad-\quad-\quad-\quad-$

PIk1-WT-GFP $(\mu \mathrm{g}) \quad-\quad 0 \quad 0.3 \quad 0.6 \quad 1.0$

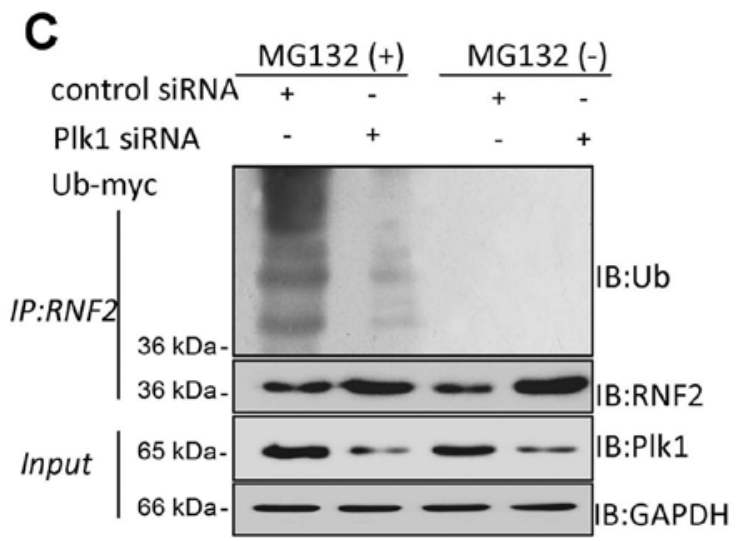

B

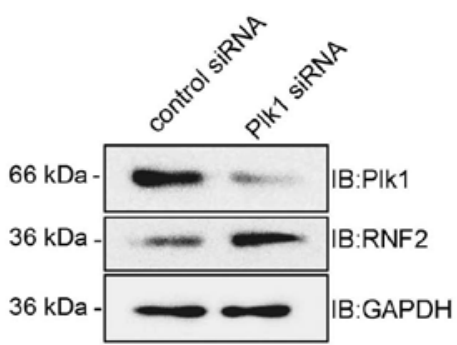

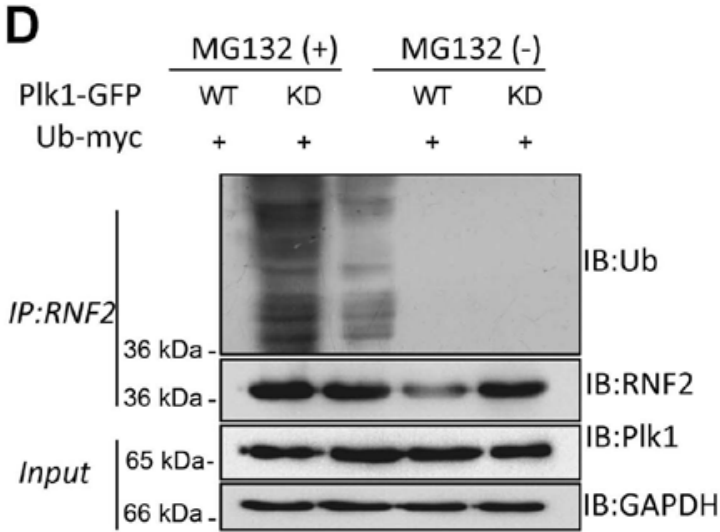

Figure 4. Plk1 interacts with RNF2 to mediate its proteasome-ubiquitin dependent degradation. (A) 293T cells were transiently co-transfected with the indicated volumes of the GFP-tagged wild-type Plk1 expression vectors, and then the mRNA levels of RNF2 were detected by qRT-PCR. (B) 293T cells were transfected with either control siRNA or siRNA targeting Plk1 for $24 \mathrm{~h}$. Then, the cell lysates were prepared and analyzed by western blot analysis using antibodies against the indicated proteins. GAPDH was included as a loading control. (C) 293T cells were transfected with either control siRNA or siRNA targeting Plk1 with myc-tagged ubiquitin for $24 \mathrm{~h}$. After treatment with or without MG132 $(10 \mu \mathrm{M})$ for $4 \mathrm{~h}$, the cells were harvested for immunoprecipitation (IP) and ubiquitination was detected by immunoblotting (IB). (D) 293T cells were transfected either with Plk1-WT-GFP or Plk1-KD-GFP with myc-tagged ubiquitin for $24 \mathrm{~h}$. After treatment with or without MG132 $(10 \mu \mathrm{M})$ for $4 \mathrm{~h}$, the cells were harvested for immunoprecipitation and ubiquitination was shown by immunoblotting. 
such as cdh1, HSF1 and Bora, and promotes their degradation by $\mathrm{SCF}^{\beta \text {-TRCP }}$. Additionally, Plk1 also phosphorylates Emi1 and promotes its degradation during mitosis. All of these results suggest that Plk1 often collaborates with $\beta$-TRCP and the SCF complex to promote protein degradation (23-25).

Abundant clinical and pathological evidence indicates that RNF2 is expressed at high levels in several cancer types compared with normal tissues $(15,26)$. Furthermore, RNF2 negatively regulates autophagy by promoting AMBRA1 degradation (27). All of these data imply that RNF2 could be considered a potential biomarker and therapeutic target for these cancer types. However, it remains elusive as to whether RNF2 is involved in mitotic progression. During mitotic division, the multifunctionality of Plk1 is relevant to its dynamic subcellular localization $(3,28,29)$. Plk1 is primarily located in the centrosome and kinetochore during prophase, and then translocates to the spindle intermediate region during meta-anaphase. Plk1 is concentrated at the equatorial plate during cytokinesis $(30,31)$. In the present study, we provide evidence for the interaction between RNF2 and Plk1 through co-immunoprecipitation, GST pull-down and yeast two-hybrid assays. Immunofluorescence studies showed co-localization of RNF2 and Plk1 at mitotic chromosomes during the prometaphase and metaphase. Plk1 kinase activity is required for entry into mitosis during the normal cell cycle. Some published results reveal that Plk1 is a key mediator of mitotic checkpoint inactivation, as cells that cannot activate Plk1 fail to properly dismantle the DNA damage checkpoint during mitosis and instead show DNA damage-induced Chk2 kinase activation (32). Plk1 is also involved in stabilizing kinetochore-microtubule attachments, whereas these attachments are more stable when kinetochore Plk1 levels decrease dramatically during metaphase (33). Furthermore, one study showed that Plk1 interacts with and phosphorylates LSD1 at Ser126 and this phosphorylation promotes release of LSD1 from chromatin during mitosis (34). The Plk1 KD is essential for creating the phosphorylated site that is bound by the PBD (35). Additionally, the Plk1 KD contains a nuclear localization sequence that promotes access to the nucleus by Plk1 before nuclear envelope breakdown (36). Inactivation or depletion of Plk1 after microinjection of the antibody, dominant-negative mutants, small interfering RNAs, or antisense oligonucleotides leads to defects in centrosomal maturation, mitotic failure and increased apoptosis $(3,37)$.

Some studies have revealed that RNF2 is degraded by the ubiquitin system independent of its own ubiquitin ligase activity in a process mediated by an exogenous, yet unidentified E3; however, a clear mechanism is unknown (13). Our results confirmed that Plk1 is associated with RNF2, and that Plk1 degraded RNF2 via a ubiquitin-dependent degradation pathway. Kinase-deficient Plk1 formed a more stable interaction with RNF2 compared with wild-type Plk1, suggesting that kinase-deficient Plk1 disabled the induction of RNF2 degradation. Given that expression of RNF2 is upregulated in many tumors, we speculate that the high expression of RNF2 in tumors may be a consequence of deficient Plk1 with an inability to degrade RNF2.

The present study has some limitations. First, the clinical pathological sections should be used to clarify the relationship between Plk1 and RNF2 in tumors. Second, the possibility that $\beta$-TRCP and SCF complex may be involved in Plk1-induced
RNF2 degradation was not investigated. Finally, the relationship between Plk1 and RNF2 could not be fully elucidated based on the experiments that were performed.

Taken together, for the first time, our observations demonstrated that Plk1 directly interacts with RNF2 and degrades RNF2 via the ubiquitin-dependent degradation pathway. We conclude that RNF2 may be used as a new target for mitotic regulation and tumorigenesis.

\section{Acknowledgements}

Not applicable.

\section{Funding}

The present study was supported by the National Natural Science Foundation of China (no. 81672046 to JD), the National Natural Science Foundation of Anhui Province (no. 1708085MH190 to LC), the Academic and Technology Leaders reserve candidate Fund of Anhui Province (no. 2016H080 to JD), and the Key Project of Outstanding Young Talent Support Program in Anhui Province University (no. gxyqZD2016047 to JD).

\section{Availability of data and materials}

The datasets used and/or analyzed during the current study are available from the corresponding author upon reasonable request.

\section{Authors' contributions}

JD and LJC conceived and designed the study, and critically revised the manuscript. RA and LC performed the experiments, analyzed the data and drafted the manuscript. JD, LJC, RA and LC participated in the analysis and interpretation of data. All authors read and approved the manuscript and agree to be accountable for all aspects of the research in ensuring that the accuracy or integrity of any part of the work are appropriately investigated and resolved.

\section{Ethics approval and consent to participate}

Ethical permission was obtained from the Institutional Review Board (IRB) of the Institute of Biomedicine at Anhui Medical University (Permit Number: AMU 26-093628), which records and regulates all research activities in the school. The IRB of the Anhui Medical University approved both animals and humans protocols. The approval from the IRB includes the permission of using mouse under euthanasia and all experimental procedures carried out in strict accordance with the recommendations in the Guide for the Care and Use of Laboratory Animals of the National Institutes of Health.

\section{Consent for publication}

Not applicable.

\section{Competing interests}

The authors declare that they have no competing interests. 


\section{References}

1. Zhang Z, Chen C, Cui P, Liao Y, Yao L, Zhang Y, Rui R and Ju S: Plk1 inhibition leads to a failure of mitotic division during the first mitotic division in pig embryos. J Assist Reprod Genet 34: 399-407, 2017

2. Liu $\mathbf{J}$ and Zhang $\mathrm{C}$ : The equilibrium of ubiquitination and deubiquitination at PLK1 regulates sister chromatid separation. Cell Mol Life Sci 74: 2127-2134, 2017.

3. Liu D, Davydenko O and Lampson MA: Polo-like kinase-1 regulates kinetochore-microtubule dynamics and spindle checkpoint silencing. J Cell Biol 198: 491-499, 2012.

4. Archambault V, Lépine G and Kachaner D: Understanding the polo kinase machine. Oncogene 34: 4799-4807, 2015.

5. Chen F, Zhuo X, Qin T, Guo X, Zhang C and Lai L: Designed inhibitor for nuclear localization signal of polo-like kinase 1 induces mitotic arrest. Chem Biol Drug Des 89: 732-740, 2017.

6. Jang YJ, Ma S, Terada Y and Erikson RL: Phosphorylation of threonine 210 and the role of serine 137 in the regulation of mammalian polo-like kinase. J Biol Chem 277: 44115-44120, 2002.

7. Gheghiani L, Loew D, Lombard B, Mansfeld J and Gavet O: PLK1 activation in late G2 sets up commitment to mitosis. Cell Rep 19: 2060-2073, 2017.

8. Kishi K, van Vugt MA, Okamoto K, Hayashi Y and Yaffe MB: Functional dynamics of Polo-like kinase 1 at the centrosome. Mol Cell Biol 29: 3134-3150, 2009.

9. Sumara I, Giménez-Abián JF, Gerlich D, Hirota T, Kraft C, de la Torre C, Ellenberg J and Peters JM: Roles of polo-like kinase 1 in the assembly of functional mitotic spindles. Curr Biol 14: 1712-1722, 2004

10. Carmena M, Pinson X, Platani M, Salloum Z, Xu Z, Clark A, Macisaac F, Ogawa H, Eggert U, Glover DM, et al: The chromosomal passenger complex activates Polo kinase at centromeres. PLoS Biol 10: e1001250, 2012.

11. Bentley ML, Corn JE, Dong KC, Phung Q, Cheung TK and Cochran AG: Recognition of UbcH5c and the nucleosome by the Bmil/Ring1b ubiquitin ligase complex. EMBO J 30: 3285-3297, 2011.

12. Qian T, Lee JY, Park JH, Kim HJ and Kong G: Id1 enhances RING1b E3 ubiquitin ligase activity through the Mel-18/Bmi-1 polycomb group complex. Oncogene 29: 5818-5827, 2010.

13. Ben-Saadon R, Zaaroor D, Ziv T and Ciechanover A: The polycomb protein Ring1B generates self atypical mixed ubiquitin chains required for its in vitro histone H2A ligase activity. Mol Cell 24: 701-711, 2006.

14. Wen W, Peng C, Kim MO, Ho Jeong C, Zhu F, Yao K, Zykova T, Ma W, Carper A, Langfald A, et al: Knockdown of RNF2 induces apoptosis by regulating MDM2 and p53 stability. Oncogene 33: 421-428, 2014.

15. Su WJ, Fang JS, Cheng F, Liu C, Zhou F and Zhang J: RNF2/Ring $1 \mathrm{~b}$ negatively regulates $\mathrm{p} 53$ expression in selective cancer cell types to promote tumor development. Proc Natl Acad Sci USA 110: 1720-1725, 2013.

16. Wei M, Jiao D, Han D, Wu J, Wei F, Zheng G, Guo Z, Xi W, Yang F, Xie P, et al: Knockdown of RNF2 induces cell cycle arrest and apoptosis in prostate cancer cells through the upregulation of TXNIP. Oncotarget 8: 5323-5338, 2017.

17. Qu C and Qu Y: Down-regulation of salt-inducible kinase 1 (SIK1) is mediated by RNF2 in hepatocarcinogenesis. Oncotarget 8: 3144-3155, 2017.

18. Du J, An R, Chen L, Shen Y, Chen Y, Cheng L, Jiang Z, Zhang A, Yu L, Chu D, et al: Toxoplasma gondii virulence factor ROP18 inhibits the host NF- $\kappa \mathrm{B}$ pathway by promoting p65 degradation. J Biol Chem 289: 12578-12592, 2014.

19. Zhen CY, Duc HN, Kokotovic M, Phiel CJ and Ren X: Cbx2 stably associates with mitotic chromosomes via a PRC2- or PRC1-independent mechanism and is needed for recruiting PRC1 complex to mitotic chromosomes. Mol Biol Cell 25: 3726-3739, 2014.
20. Zhang Y, Li X, Chen Z and Bepler G: Ubiquitination and degradation of ribonucleotide reductase $\mathrm{M} 1$ by the polycomb group proteins RNF2 and Bmil and cellular response to gemcitabine. PLoS One 9: e91186, 2014.

21. Steffen PA, Fonseca JP, Gänger C, Dworschak E, Kockmann T, Beisel C and Ringrose L: Quantitative in vivo analysis of chromatin binding of Polycomb and Trithorax group proteins reveals retention of ASH1 on mitotic chromatin. Nucleic Acids Res 41: 5235-5250, 2013

22. Fonseca JP, Steffen PA, Müller S, Lu J, Sawicka A, Seiser C and Ringrose L: In vivo Polycomb kinetics and mitotic chromatin binding distinguish stem cells from differentiated cells. Genes Dev 26: 857-871, 2012.

23. Fukushima H, Ogura K, Wan L, Lu Y, Li V, Gao D, Liu P, Lau AW, Wu T, Kirschner MW, et al: SCF-mediated Cdh1 degradation defines a negative feedback system that coordinates cell-cycle progression. Cell Rep 4: 803-816, 2013.

24. Lee YJ, Kim EH, Lee JS, Jeoung D, Bae S, Kwon SH and Lee YS: HSF1 as a mitotic regulator: Phosphorylation of HSF1 by Plk1 is essential for mitotic progression. Cancer Res 68: 7550-7560, 2008.

25. Seki A, Coppinger JA, Du H, Jang CY, Yates JR III and Fang G: Plk1- and beta-TrCP-dependent degradation of Bora controls mitotic progression. J Cell Biol 181: 65-78, 2008.

26. Li XQ, He WP, Hou WH, Chen JW, Fan RR, Yuan LJ, Yang GP, Cai MY, Chen L, Li J, et al: Overexpression of RNF2 is positively associated with ovarian carcinoma aggressiveness and indicative of poor patient survival. Oncotarget 6: 31181-31190, 2016.

27. Xia P, Wang S, Huang G, Du Y, Zhu P, Li M and Fan Z: RNF2 is recruited by WASH to ubiquitinate AMBRA1 leading to downregulation of autophagy. Cell Res 24: 943-958, 2014.

28. Lera RF, Potts GK, Suzuki A, Johnson JM, Salmon ED, Coon JJ and Burkard ME: Decoding Polo-like kinase 1 signaling along the kinetochore-centromere axis. Nat Chem Biol 12: 411-418, 2016.

29. Lu LY and Yu X: The balance of Polo-like kinase 1 in tumorigenesis. Cell Div 4: 4, 2009.

30. Wachowicz P, Fernández-Miranda G, Marugán C, Escobar B and de Cárcer G: Genetic depletion of Polo-like kinase 1 leads to embryonic lethality due to mitotic aberrancies. Bioessays 38 (Suppl 1): S96-S106, 2016.

31. Hasegawa H, Hyodo T, Asano E, Ito S, Maeda M, Kuribayashi H, Natsume A, Wakabayashi T, Hamaguchi M and Senga T: The role of PLK1-phosphorylated SVIL in myosin II activation and cytokinetic furrowing. J Cell Sci 126: 3627-3637, 2013.

32. van Vugt MA, Gardino AK, Linding R, Ostheimer GJ, Reinhardt HC, Ong SE, Tan CS, Miao H, Keezer SM, Li J, et al: A mitotic phosphorylation feedback network connects Cdk1, Plk1, 53BP1, and Chk2 to inactivate the $\mathrm{G}_{2} / \mathrm{M}$ DNA damage checkpoint. PLoS Biol 8: e1000287, 2010.

33. Lénárt P, Petronczki M, Steegmaier M, Di Fiore B, Lipp JJ, Hoffmann M, Rettig WJ, Kraut N and Peters JM: The small-molecule inhibitor BI 2536 reveals novel insights into mitotic roles of polo-like kinase 1. Curr Biol 17: 304-315, 2007.

34. Peng B, Shi R, Jiang W, Ding YH, Dong MQ, Zhu WG and $\mathrm{Xu}$ X: Phosphorylation of LSD1 by PLK1 promotes its chromatin release during mitosis. Cell Biosci 7: 15, 2017.

35. Park JE, Soung NK, Johmura Y, Kang YH, Liao C, Lee KH, Park CH, Nicklaus MC and Lee KS: Polo-box domain: A versatile mediator of polo-like kinase function. Cell Mol Life Sci 67: 1957-1970, 2010.

36. Taniguchi E, Toyoshima-Morimoto F and Nishida E: Nuclear translocation of plk1 mediated by its bipartite nuclear localization signal. J Biol Chem 277: 48884-48888, 2002.

37. Tao YF, Li ZH, Du WW, Xu LX, Ren JL, Li XL, Fang F, Xie Y, $\mathrm{Li}$ M, Qian GH, et al: Inhibiting PLK1 induces autophagy of acute myeloid leukemia cells via mammalian target of rapamycin pathway dephosphorylation. Oncol Rep 37: 1419-1429, 2017. 